\title{
MODELING, DESIGN, AND OPTIMIZATION OF A SOLID STATE ELECTRON SPIN QUBIT*
}

\author{
R. E. CAFLISCH ${ }^{\dagger}$, MARK F. GYURE ${ }^{\ddagger}$, HANS D. ROBINSON $\S$, AND \\ ELI YABLONOVITCH ${ }^{\dagger}$
}

\begin{abstract}
This paper describes a solid state system in which a qubit is realized as the spin of a single trapped electron in a quantum dot and read functionality is via an adjacent quantum wire with a single or a small number of conductive states. Because of the limited design window for this system, simulation is an important guide to an experimental search for successful designs. We use a semianalytic approximation that is accurate enough to provide meaningful results and computationally simple enough to allow high throughput, as needed for design and optimization. In particular, we find designs that achieve double pinchoff (i.e., a single trapped electron in the dot and a single conductive state in the wire). After relaxing the design requirements to allow for a small number of conductive states in the wire, we find successful designs that are optimally robust, in the sense that their success is unlikely to be affected by fabrication errors.
\end{abstract}

Key words. qubit, quantum dot, quantum computing, design, optimization

AMS subject classifications. $81 \mathrm{P} 68,35 \mathrm{P} 20$

DOI. $10.1137 / 040606181$

1. Introduction. Quantum logic, based on manipulation and interaction of binary quantum states or "qubits," has great potential for communication and computation. Quantum communication could offer absolute security [14] and transmission rates beyond the Shannon limit [5]. Quantum computation could greatly accelerate the solution of certain important problems, such as prime factorization [15], database searching [6], and simulation of quantum systems [16]. This potential has motivated a large effort to develop and implement quantum logic. Currently, the foremost problem for quantum communication and computation is the implementation of qubits in a robust and scalable system, which will allow for error correction and control of decoherence. Solid state implementations of a qubit, based on an electron or nuclear spin confined to a quantum dot, have been proposed in $[1,8,9,10,11,12,17,18]$.

This paper is concerned with the design of a single qubit system in a solid state implementation, as proposed in [3, 17], in which a qubit is represented as the spin of a single electron confined in a quantum dot. A quantum wire is placed below the quantum dot, so that the conductivity of the wire will depend sensitively on the charge present in the dot. The wire can then be used to verify the presence of a single electron in the quantum dot and, in the presence of a spin polarized electron reservoir, to read out the spin of that electron. The latter can be accomplished in several ways, for instance, by measuring tunneling times from the reservoir into the qubit. As the spin singlet state has lower energy than the triplet states [4], we can arrange to make tunneling into a triplet state energetically forbidden, and since the singlet state must

\footnotetext{
* Received by the editors April 2, 2004; accepted for publication (in revised form) October 6, 2004; published electronically April 14, 2005. This research was supported in part by a DARPA grant as part of the Quantum Information Science and Technology (QuIST) Initiative and by the Center for Scalable and Integrated Nanomanufacturing, NSF grant DMI-0327077.

http://www.siam.org/journals/siap/65-4/60618.html

${ }^{\dagger}$ Mathematics Department, UCLA, Los Angeles, CA 90095-1555 (caflisch@math.ucla.edu).

${ }^{\ddagger}$ HRL Laboratories, 3011 Malibu Canyon Road, Malibu, CA 90265 (gyure@hrl.com).

§Electrical Engineering Department, UCLA, Los Angeles, CA 90095 (hansr@ee.ucla.edu, eliy@ee. ucla.edu).
} 
be formed using two opposite spins, the tunneling time will then depend strongly on the relative alignment of the qubit spin to the reservoir spin.

In our design, the quantum dot and wire are formed in two vertically stratified, parallel semiconductor quantum wells and are defined electrostatically using lithographically patterned gates on the surface of the semiconductor. Further description of the geometry and electrostatics of this system are provided in section 2. An alternative design using a horizontal placement of quantum dots has been carried out in [18].

A successful qubit design requires a single electron in the quantum dot and a small number of conduction states in the quantum wire. If there is only a single state in the quantum wire, it can be used both as spin reservoir and charge sensor. Moreover, the design should be robust with respect to fluctuations or errors in modeling and fabrication. These are very stringent requirements that are difficult to satisfy, and numerical simulation can serve as an important guide in the experimental search for successful designs.

The principal goal of the present study is development of a semianalytic model and its application to design and optimization for this quantum system. This reduced order model is based on a number of approximations that restrict its validity. Comparison to full scale numerical simulations, however, indicate that its accuracy is sufficient to provide meaningful results. Computational speed is the model's great virtue, enabling the high throughput that is required for design and optimization of the quantum system.

There are three distinct aspects to simulation of this quantum system: construction of a mathematical model embodying the correct physics, development of an effective numerical method for solving the model, and use of the numerical method to search for a successful design. The semianalytic model and its use for design and optimization, as presented in this paper, address only the last of these. Related efforts, which are beyond the scope of this paper, include a full-scale numerical method for the Schrödinger-Poisson model [2] and simulations using nextnano ${ }^{3}[13]$ that include more detailed physics.

Furthermore, design of this qubit system is an intermediate, but important step toward the much more challenging goal of constructing a quantum device. A functioning quantum device using this qubit system must satisfy additional requirements, such as preparation of initial data, coupling of qubits, measurement of the qubit state, control of decoherence, and error correction, that are not included in the present design problem.

In section 2 we develop a Schrödinger-Poisson model for simulation of the electrostatic potential and the single electron wavefunction, and we formulate design goals for performance of this system. A reduced order, semianalytic model is derived in section 3 using square well or parabolic approximations for the electrostatic potential. In section 4 the accuracy and validity of this semianalytic model is assessed by comparison to full scale numerical solution of the Schrödinger-Poisson model from [2]. Successful designs with double pinchoff are found in section 5 through a random search in parameter space. A measure of design robustness, in terms of the sensitivity of the design to fabrication errors, is formulated in section 6 . In section 7, an analysis is presented that greatly simplifies the computation of design robustness. Using this simplified analysis, a search for designs that are optimally robust is described in section 8. Finally, conclusions are presented in section 9 .

2. Qubit design problem. This sections describes the solid state system and the design goals for a qubit. This description includes one-, two-, and three-dimensional versions of the system. 
2.1. Qubit system description. The layered semiconductor system consists of a series of material layers, with layer $i$ consisting of material $m_{i}$ and having thickness $d z_{i}$, in which $z$ is a measure of the distance from the top planar surface, increasing in the downward direction. In addition there are $\delta$-doped layers of zero thickness at the boundaries of some of the material layers, with a density $\sigma_{k}$ of ions per area in the $k$ th $\delta$-doped layer. Note that the charge density $\sigma$ is the doping density times an activation factor, so that it is less than the actual doping density. Volumetric doping, including intrinsic doping, is neglected.

As an example that will be used in this study and is pictured in Figure 1, consider a system that consists of the following layers, in order starting at $z=0$ :

- layer of material $A$ of thickness $d z_{1}$;

- $\delta$-doped layer with charge density $\sigma_{1}$;

- layer of material $A$ of thickness $d z_{2}$;

- layer of material $B$ of thickness $d z_{3}$, the layer containing the quantum dot;

- layer of material $A$ of thickness $d z_{4}$;

- layer of material $B$ of thickness $d z_{5}$, the layer containing the quantum wire;

- layer of material $A$ of thickness $d z_{6}$;

- $\delta$-doped layer with charge density $\sigma_{2}$;

- layer of material $A$ of infinite thickness.

The geometry of these layers is one-dimensional; higher dimensionality is determined by the geometry of the gates. At the top of the material system, i.e., $z=0$, there are a series of gates $G_{m}$ on which the electron potential energy $\phi_{m}$ is specified. Away from the gates, the energy is taken to be equal to a constant free surface potential $\phi_{0}$.

In this study the following gate geometries and potentials are considered:

- gate $G_{g}$ consisting of a circle $r<R_{g} / 2$ for the three-dimensional geometry or an interval $|x|<R_{g} / 2$ for the two-dimensional geometry, on which the potential energy is $\phi_{g}$;

- two gates $G_{b \pm}$, in which $G_{b+}$ consists of points with $x>R_{b} / 2$ and $G_{b-}$ consists of points with $x<-R_{b} / 2$ in both the two-dimensional and threedimensional geometries, with potential $\phi_{b}$ on both gates;

- no gates for the one-dimensional geometry;

- potential energies $\phi_{g}=-V_{g}+\phi_{\text {schottky }}$ and $\phi_{b}=-V_{b}+\phi_{\text {schottky, where }}$ $\phi_{\text {schottky }}$ is the Schottky barrier, and $V_{g}$ and $V_{b}$ are the voltages applied to the gates.

In this description all distances are measured in $n m$, the doping densities are measured in units of electrons $\mathrm{cm}^{-2}$, and the energy $\phi$ is in units of $\mathrm{eV}$.

A drawing of the device structure, with parameters from an optimally robust design as in (8.1), is shown in Figure 1. A schematic drawing of the gates, the potentials in the quantum wells, and the electron densities is shown in Figure 2. Positive potential energy $\left(\phi_{b}\right)$ on the planar side gates raises the potential on the sides of both quantum wells. Negative potential energy $\left(\phi_{g}\right)$ on the circular, central gates lowers the potential energy, primarily in the upper well. This leads to a localized electron density (i.e., a quantum dot) in the upper well and an electron density along a line (i.e., a quantum wire) in the lower quantum well.

The layer widths $d z_{i}$ and the charge densities $\sigma_{i}$ are determined during the material growth, and the gate sizes $R_{i}$ are determined during the device fabrication. These parameters cannot be changed after fabrication. Thus the parameters can be divided into two sets: the vector of operation parameters $v_{o}=\left(\phi_{g}, \phi_{b}\right)$, which can be varied during operation of the device, and the vector of design parameters 


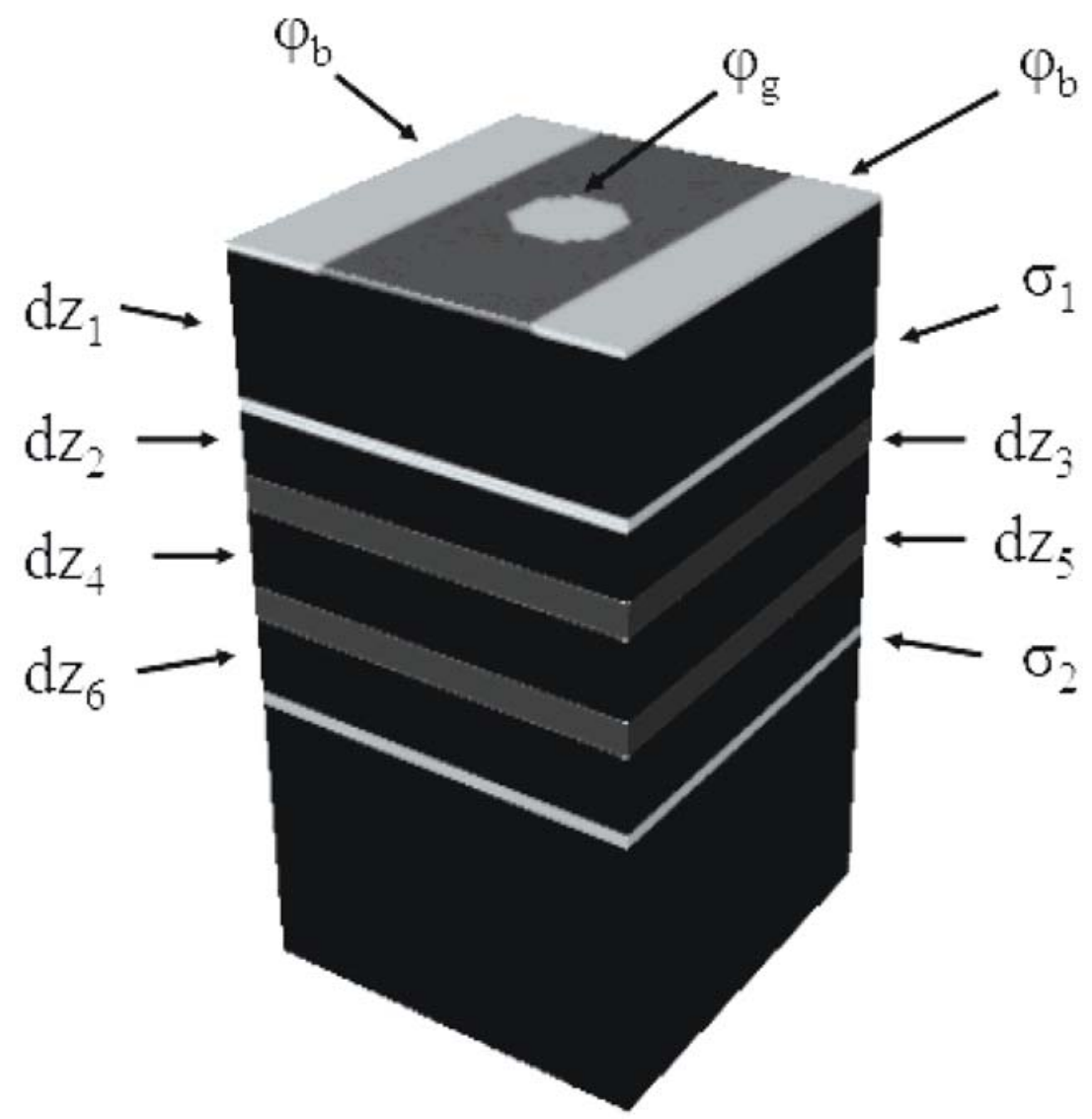

FIG. 1. Schematic drawing of the device geometry. The depths of the material layers are $d z_{i}$, in which $d z_{3}$ is the depth of the upper quantum well, which contains the quantum dot, and $d z_{5}$ is the depth of the lower quantum well, which contains the quantum wire. The charge densities in the delta-doped layers are $\sigma_{i}$. The electrostatic potential of the central gate (a circle of diameter $R_{g}$ in a three-dimensional geometry) is $\phi_{g}$, and the electrostatic potential of the two side gates (separated by a distance $R_{b}$ ) is $\phi_{b}$. In a two-dimensional geometry the central gate would be an infinite strip parallel to the side gates.

$v_{d}=\left(d z_{1}, d z_{2}, d z_{3}, d z_{4}, d z_{5}, d z_{6}, R_{g}, R_{b}, \sigma_{1}, \sigma_{2}\right)$, which cannot be changed during operation. A device design can be identified with a choice of the design vector $v_{d}$. These are chosen from a subset $C$ of $R^{10}$ that has been determined from some external consideration, such as additional constraints or previous experience. These have the form

$$
\begin{aligned}
\underline{d z}_{i} & <d z_{i}<\overline{d z}_{i} \text { for } 1 \leq i \leq 6, \\
\underline{R}_{g} & <R_{g}<\bar{R}_{g} \\
\underline{R}_{b} & <R_{b}<\bar{R}_{b} \\
\underline{\sigma}_{i} & <\sigma_{i}<\bar{\sigma}_{i} \text { for } 1 \leq i \leq 2, \\
\underline{\phi}_{g} & <-\phi_{g}<\bar{\phi}_{g} \\
\underline{\phi}_{b} & <\phi_{b}<\bar{\phi}_{b} .
\end{aligned}
$$



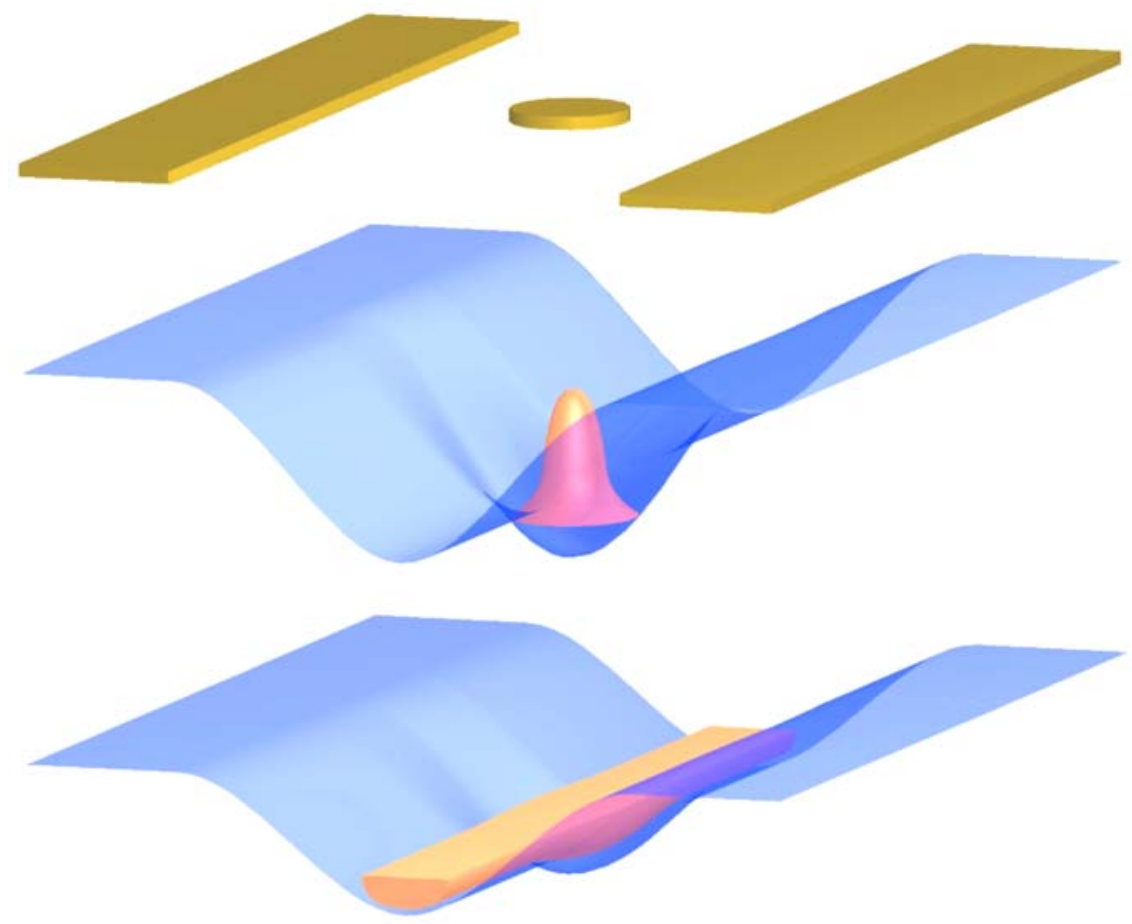

FIG. 2. Schematic drawing of the gates, the potential energies in the quantum wells, and the electron densities in the dot and the wire.

In the design searches conducted below, a typical set of constraints was the following:

$$
\begin{aligned}
20< & d z_{1}<40(\mathrm{~nm}), \\
20< & d z_{2}<40(\mathrm{~nm}), \\
5< & d z_{3}<10(\mathrm{~nm}), \\
& \quad d z_{4}=16(\mathrm{~nm}), \\
5< & d z_{5}<15(\mathrm{~nm}), \\
& d z_{6}=50(\mathrm{~nm}), \\
50< & R_{g}<100(\mathrm{~nm}) \\
50< & \left(R_{b}-R_{g}\right) / 2<400(\mathrm{~nm}), \\
0< & \sigma_{1}<4 \times 10^{11}\left(\mathrm{~cm}^{-2}\right), \\
0< & \sigma_{2}<4 \times 10^{11}\left(\mathrm{~cm}^{-2}\right), \\
0.1< & -\phi_{g}<0.3(\mathrm{eV}) \\
0< & \phi_{b}<2.0(\mathrm{eV}) .
\end{aligned}
$$

The constraints on $d z_{4}$ were chosen to allow electrons to tunnel between dot and wire on a ms time scale; $16 \mathrm{~nm}$ is appropriate for the $\mathrm{InP} / \mathrm{InGaAs}$ system. The lower bounds on $R_{g}$ and $R_{b}$ are representative of what can be easily accomplished with e-beam lithography. The upper bounds on $\sigma_{i}$ are set to avoid hopping conduction through the doping layers. The upper bound on $\phi_{b}$ and the lower bound on $\phi_{g}$ are set 
to avoid passing any current through the gates into the sample, which would disrupt the qubit. The lower bound on $\phi_{g}$ is set to ensure formation of a quantum dot with a bound electron state; at smaller voltages, the electron may not be bound, which the central gate, the parabolic approximation in section 3 may fail to predict. The remaining constraints are reasonable limits that were imposed to speed the design search.

2.2. Schrödinger-Poisson model. The electrostatic potential $\Phi$ is assumed to satisfy the Poisson equation

$$
\nabla \cdot \epsilon \nabla \Phi=\sigma_{1} \delta_{1}+\sigma_{2} \delta_{2}-\rho_{\psi}
$$

in which $\epsilon=k \epsilon_{0} / e^{2}$ is the scaled dielectric constant, $\delta_{i}$ is a $\delta$-function on $i$ th $\delta$ doped interface, and $\rho_{\psi}$ is the number density for electrons in the wire, which must be determined self-consistently as described below. The function $\Phi$ is the potential energy for an electron, measured in $\mathrm{eV}$. For this equation the boundary conditions are taken to be Dirichlet conditions (i.e., $\Phi$ prescribed) at the top, Neumann conditions (i.e., $\partial \Phi / \partial n=0$ ) at the bottom, and periodic conditions on the sides.

The electronic wave function for the unbound electron of lowest energy is assumed to satisfy a single particle Schrödinger equation

$$
-\nabla \cdot\left(\frac{\hbar^{2}}{2 m} \nabla \Psi\right)=-(\Phi+U) \Psi+\lambda \Psi
$$

in which $e$ is the electron charge, $m$ is the electron effective mass, $U$ is the conduction band offset relative to material $A$ (InP in the examples below), $\lambda$ is the energy level (eigenvalue) in units of $e V$, and $\Psi$ is wave function (eigenfunction).

As an example, for $\operatorname{InP}, I n_{x} G a_{1-x} A s$, and $A l_{y} I n_{1-y} A s$, with alloy fractions $x=0.53$ and $y=0.48$, the material parameters are given in Table 1 and the relevant physical constants are given in Table 2 .

TABLE 1

Material parameters.

\begin{tabular}{||l|llll||}
\hline Parameter & InP & $I n_{0.53} G a_{0.48} A s$ & $A l_{0.48} I n_{0.52} A s$ & Units \\
$k$ & 12.61 & 13.9 & 12.7 & 1 \\
$\epsilon=k \epsilon_{0} / e^{2}$ & .697 & .769 & 0.702 & $1 /(\mathrm{eV} \mathrm{nm})$ \\
$m$ & .079 & .041 & 0.0733 & $m_{0}$ \\
$\hbar^{2} / 2 m$ & .484 & .94 & 0.522 & $\mathrm{eV} / \mathrm{nm}^{2}$ \\
$U$ & 0 & .224 & 0.25 & $\mathrm{eV}$ \\
\hline
\end{tabular}

TABLE 2

Physical constants.

\begin{tabular}{||l|ll||}
\hline Constant & Value & Units \\
\hline$\hbar^{2} / 2 m_{0}$ & .0382 & $\mathrm{eV} / \mathrm{nm}^{2}$ \\
$\epsilon_{0}$ & $8.854 \times 10^{-12}$ & $\mathrm{C}^{2} N^{-1} \mathrm{~m}^{-2}$ \\
$\epsilon_{0} / \mathrm{e}^{2}$ & .0553 & $1 /(\mathrm{eV} \mathrm{nm})$ \\
\hline
\end{tabular}

For the solutions of interest in this study, the electrons are localized either in a dot in the upper layer or along a wire in the lower layer. This implies that the eigenfunctions for the Schrödinger equation (2.9) are each localized in either the dot or the wire. The eigenvalues and eigenfunctions in the quantum dot are labeled 
$\lambda_{k}^{d}, \Psi_{k}^{d}$; those in the wire are labeled $\lambda_{k}^{w}, \Psi_{k}^{w}$. Also denote $d \lambda=\lambda_{2}-\lambda_{1}$ as the difference between the first two eigenvalues. The eigenfunctions are normalized so that $\int|\Psi|^{2} d x=1$. The self-consistent charge density is $\rho_{\psi}=\sum|\Psi|^{2}$, summed over all $\lambda<E_{F}$, in which the Fermi energy $E_{F}$ is set to 0 .

To emphasize the dependence of the eigenvalues $\lambda$ on the gate voltages $\phi_{g}$ and $\phi_{b}$ and the design vector $v$, we shall sometimes write $\lambda=\lambda\left(\phi_{g}, \phi_{b}, v\right)$.

Note that the eigenfunctions for the quantum dot are quite distinct from those for the quantum wire. So computation of these eigenfunctions is equivalent to a computation using two separate wave functions for the dot and wire, and so it correctly represents the charge density in the wire and its affect on the dot. Interaction terms between the dot and wire are omitted, because they are small. On the other hand, tunneling effects between the dot and wire are important for detection of an electron in the dot using the wire. These tunneling effects are beyond the scope of the current model.

2.3. Design goals. The design goals for the quantum dot are to have a single confined electron under the gate and no confined electron states away from the gate. The design goals for the quantum wire are to have a small number $k$ of conduction states in the wire, with no additional states in the wire under the gate. Denote $E_{k}^{d}$ and $E_{k}^{w}$ to be the energy for $k$ electrons in the dot and for $k$ conduction states in the wire, respectively. Also denote $E_{k}^{d}(0)$ and $E_{k}^{w}(0)$ to be the same energies but with no voltage on the central gate; i.e., $\phi_{g}=0$. An unbound electron will be localized if its energy is less than the Fermi energy $E_{F}=0$. The design goals can thus be stated as

$$
\begin{aligned}
E_{1}^{d} & <0<E_{2}^{d}, \\
0 & <E_{1}^{d}(0), \\
E_{k}^{w} & <0<E_{k+1}^{w}, \\
E_{k}^{w}(0) & <0<E_{k+1}^{w}(0) .
\end{aligned}
$$

Since $E_{k}^{w}<E_{k}^{w}(0),(2.12)$, and (2.13) can be recombined as

$$
E_{k}^{w}(0)<0<E_{k+1}^{w} .
$$

As shown below, the energy level $E_{2}^{d}$ is smaller than $E_{1}^{d}(0)$ in the regime of interest, so that (2.11) is redundant.

In the quantum dot, the energy for a single electron is the lowest eigenvalue, so that $E_{1}^{d}=\lambda_{1}^{d}$ and $E_{1}^{d}(0)=\lambda_{1}^{d}(0)$. For the energy of two electrons, there is an interaction (Coulomb) correction $E_{2}^{d}=\lambda_{2}^{d}+\tilde{E}_{2}^{d}$. In the wire, we identify a conduction state, as an eigenfunction for the cross-section of the wire and neglect the interaction among different conduction states. Thus $E_{k}^{w}=\lambda_{k}^{w 2 D}$ and $E_{k}^{w}(0)=\lambda_{k}^{w 2 D}(0)$, in which $\lambda_{k}^{w 2 D}$ and $\lambda_{k}^{w 2 D}(0)$ are the two-dimensional eigenvalues and $\phi_{g}=0$ for $\lambda_{k}^{w 2 D}(0)$. Therefore the operation goals can be rewritten as

$$
\begin{aligned}
\lambda_{1}^{d} & <0<\lambda_{2}^{d}+\tilde{E}_{2}^{d}, \\
\lambda_{1}^{w 2 D}(0) & <0<\lambda_{2}^{w 2 D} .
\end{aligned}
$$

The design goal is to find a device design $v_{d}$, for which there is a choice of operation parameters $v_{o}$ such that the operation goals (2.15) and (2.16) are satisfied. A second design goal, that the operation goals are still met in the presence of growth and fabrication uncertainties, is formulated in section 6 . 
3. Semianalytic model. In this section we formulate a simplified semianalytic model that represents an approximate solution of the Schrödinger-Poisson equation. As described below, the potential $\Phi$ in each of the upper and lower quantum wells is approximated as a parabola in the lateral directions $x$ and $y$ and a square well in the depth direction $z$. Because the layered geometry is independent of $x$ and $y$ and the gates have a reflection symmetry with respect to both $x$ and $y$, the first derivatives $\Phi_{x}$ and $\Phi_{y}$ are 0 on the centerline $x=y=0$. Thus the lateral variation of the potential near the either quantum dot and quantum wire is approximately given by $\frac{1}{2}\left(x^{2} \Phi_{x x}+y^{2} \Phi_{y y}\right)$.

3.1. Approximations for electrostatics. For the potential $\Phi=\Phi^{1 D}$ due to modulationally doped layers but not including the effect of the gates, put the bottom boundary condition at $\infty$, omit any self-consistent terms, and neglect the variation in dielectric constant by using the value for material A throughout to obtain

$$
\Phi^{1 D}= \begin{cases}\phi_{\text {top }}-\epsilon_{A}^{-1}\left(\sigma_{1}+\sigma_{2}\right) z, & 0<z<z_{1}, \\ \phi_{\text {top }}-\epsilon_{A}^{-1}\left(\sigma_{1} z-\sigma_{2} z_{1}\right), & z_{1}<z<z_{2}, \\ \phi_{\text {top }}-\epsilon_{A}^{-1}\left(\sigma_{1} z_{2}-\sigma_{2} z_{1}\right), & z_{2}<z,\end{cases}
$$

in which $z_{1}=d z_{1}$ and $z_{2}=d z_{1}+d z_{2}+d z_{3}+d z_{4}+d z_{5}+d z_{6}$ are the positions of the $\delta$-doped layers.

The potential $\Phi=\Phi_{L}^{2 D}$, due to a gate that is a strip (in three dimensions) (i.e., $|x|<L / 2, z=0$ ) with potential $\Phi=1$ on the gate and $\Phi=0$ away from gate, is

$$
\Phi_{L}^{2 D}(x, z)=\pi^{-1}\left(\arctan \left(\frac{x+L / 2}{z}\right)-\arctan \left(\frac{x-L / 2}{z}\right)\right) .
$$

On the central axis $x=0$, the values of $\Phi$ and its second derivative are

$$
\begin{gathered}
\Phi_{L}^{2 D}(x=0, z)=2 \pi^{-1} \arctan (L / 2 z), \\
\Phi_{L x x}^{2 D}(x=0, z)=-\pi^{-1} z^{-2} \frac{2 L / z}{\left(1+L^{2} / 4 z^{2}\right)^{2}} .
\end{gathered}
$$

The potential $\Phi=\Phi_{d}^{3 D}$, due to a gate that is a circle (i.e., $r=|(x, y)|<d / 2$ ) with potential $\Phi=1$ on the gate and $\Phi=0$ away from gate, is

$$
\begin{aligned}
\Phi_{d}^{3 D}(\mathbf{x}) & =\Phi(r, z) \\
& =\frac{|z|}{2 \pi} \int_{0}^{2 \pi} \int_{0}^{d / 2}\left|\mathbf{x}-\mathbf{x}^{\prime}\right|^{-3} r^{\prime} d r^{\prime} d \theta^{\prime} \\
& \left.=\frac{|z|}{2 \pi} \int_{0}^{2 \pi} \int_{0}^{d / 2}\left(z^{2}+\left(r-r^{\prime} \cos \theta^{\prime}\right)^{2}+r^{\prime 2} \sin ^{2} \theta^{\prime}\right)^{2}\right)^{-3 / 2} r^{\prime} d r^{\prime} d \theta^{\prime} .
\end{aligned}
$$

On the central axis $r=0$, the values of $\Phi$ and its second derivative are

$$
\begin{aligned}
\Phi_{d}^{3 D}(r=0, z) & =1-\left(1+(d / 2 z)^{2}\right)^{-1 / 2} \\
\Phi_{d r r}^{3 D}(r=0, z) & =-\frac{3}{2}|z|(d / 2)^{2}\left(z^{2}+(d / 2)^{2}\right)^{-5 / 2} .
\end{aligned}
$$

Add these together to obtain the total potential as

$$
\Phi= \begin{cases}\Phi^{1 D}+\phi_{b}\left(1-\Phi_{d}^{2 D}\right)+\phi_{g} \Phi_{L}^{2 D} & \text { in two dimensions } \\ \Phi^{1 D}+\phi_{b}\left(1-\Phi_{d}^{2 D}\right)+\phi_{g} \Phi_{L}^{3 D} & \text { in three dimensions. }\end{cases}
$$


The second derivatives of the total potential on the central axis are

$$
\begin{aligned}
& \Phi_{x x}= \begin{cases}-\phi_{b} \Phi_{d x x}^{2 D}+\phi_{g} \Phi_{L x x}^{2 D} & \text { in two dimensions, } \\
-\phi_{b} \Phi_{d x x}^{2 D}+\phi_{g} \Phi_{L r r}^{3 D} & \text { in three dimensions, }\end{cases} \\
& \Phi_{y y}= \begin{cases}0 & \text { in two dimensions, } \\
\phi_{g} \Phi_{L r r}^{3 D} & \text { in three dimensions. }\end{cases}
\end{aligned}
$$

All the subsequent computations for the semianalytic model were performed using MATLAB programs.

3.2. Approximations for Schrödinger. The approximation for the Schrödinger eigenfunctions and eigenvalues relies on separation of variables: if $m$ is constant and $\Phi(x, y, z)=\Phi^{x}(x)+\Phi^{y}(y)+\Phi^{z}(z)$, then

$$
\begin{aligned}
\lambda & =\lambda^{x}+\lambda^{y}+\lambda^{z}, \\
\Psi(x, y, z) & =\Psi^{x}(x) \Psi^{y}(y) \Psi^{z}(z)
\end{aligned}
$$

in which

$$
\begin{aligned}
& -\left(\hbar^{2} / 2 m\right) \Psi_{x x}^{x}=-\Phi^{x} \Psi^{x}+\lambda^{x} \Psi^{x}, \\
& -\left(\hbar^{2} / 2 m\right) \Psi_{y y}^{y}=-\Phi^{y} \Psi^{y}+\lambda^{y} \Psi^{y}, \\
& -\left(\hbar^{2} / 2 m\right) \Psi_{z z}^{z}=-\Phi^{z} \Psi^{z}+\lambda^{z} \Psi^{z} .
\end{aligned}
$$

Use separation of variables to find eigenvalues in a channel of width $w$ and center $z$. Neglect variation of $\Phi$ across the well and approximate the $x$-dependence for $\Phi^{2 D}$ (i.e., for a gate that is a strip in three dimensions) or the $(x, y)$-dependence for $\Phi^{2 D}$ (i.e., for a gate that is a circle in three dimensions) as parabolic with

$$
\begin{array}{ll}
\Phi^{2 D} \approx \Phi_{2 D}^{x}(x) & =.5 \Phi_{x x}(x=0, z) x^{2}, \\
\Phi^{3 D} \approx \Phi_{3 D}^{x}(x)+\Phi_{3 D}^{y}(y) & =.5 \Phi_{r r}(r=0, z)\left(x^{2}+y^{2}\right) .
\end{array}
$$

Both the two-dimensional and three-dimensional problems have been written as a sum of one-dimensional parabolic potentials. The eigenvalue and eigenvalue spacing for a one-dimensional parabolic potential $\Phi(x)=\phi_{b} x^{2}$ are

$$
\begin{aligned}
\lambda_{1}^{p} & =\left(\Phi_{x x} \hbar^{2} / 4 m\right)^{1 / 2}, \\
d \lambda^{p} & =2 \lambda_{1}^{p} .
\end{aligned}
$$

We denote $\lambda^{p x}$ and $\lambda^{p y}$ for the eigenvalues due to the parabolic potential in the $\mathrm{x}$ and y-directions, respectively, and $d \lambda^{p x}$ and $d \lambda^{p y}$ for the corresponding eigenvalue spacing.

In the $z$-direction, the potential is approximately a square well, since

$$
\Phi^{z}(z)= \begin{cases}0 & \left|z-z_{0}\right|>L / 2 \\ -U & \left|z-z_{0}\right|<L / 2\end{cases}
$$

in which $U$ is the offset in wells, neglecting variation across the well. The eigenvalues for this square well are solutions of

$$
\begin{aligned}
\lambda^{s w} & =c_{1} k_{1}^{2}-U, \\
k_{1}^{2}\left(1+\left(c_{1} / c_{0}\right) \tan ^{2}\left(k_{1} w / 2\right)\right) & =U / c_{1}
\end{aligned}
$$


in which $c_{0}$ and $c_{1}$ are the values of $\hbar^{2} / 2 m$ outside the well and in the well, respectively; i.e., $c_{0}$ is the value for material $A(\mathrm{InP})$ and $c_{1}$ is the value for material $B$ (InGaAs).

In summary, the eigenfunctions, lowest eigenvalue and eigenvalue spacing are

$$
\begin{aligned}
\Psi & =\Psi^{s w}(z) \Psi^{p x}(x) \Psi^{p y}(y), \\
\lambda & =\lambda^{s w}+\lambda^{p x}+\lambda^{p y}, \\
d \lambda & = \begin{cases}d \lambda^{p x} & \text { in two dimensions, } \\
\min \left(d \lambda^{p x}, d \lambda^{p y}\right) & \text { in three dimensions. }\end{cases}
\end{aligned}
$$

In the simplest model, we also take the energy for two electrons to be the same as the second eigenvalue in the quantum dot; i.e., set $\tilde{E}_{2}^{d}=0$ in (2.15).

3.3. Generalizations. Two generalizations of the semianalytic model of the previous section are formulated here to include effects of Coulomb interactions and self-consistent terms.

An approximation to the Coulomb correction $\tilde{E}_{2}^{d}$ for two electrons in the quantum dot has been developed by Gyure [7]. He computed the energy for two electrons in a one-dimensional parabolic potential using an iterative projection method, then fit the result to the following simple formula:

$$
E_{2}^{d}=\lambda_{1}^{d}+c r_{d} \gamma_{y}^{\kappa}
$$

in which $r_{d}=0.00289 \mathrm{eV}$ is the Rydberg energy, $\gamma_{y}=\lambda_{1}^{d}$, and the (dimensionless) fitting parameters are $c=3.5213$ and $\kappa=0.75654$. The one-dimensional approximation was justified by two-dimensional calculations that showed the anisotropy of the potential is large enough in most cases to ignore the smaller dimension. The error induced is relatively small and decreases rapidly with anisotropy ratio.

The most significant self-consistent terms are the effect of the charge in the wire on the potential in the dot. For a wire defined by a parabolic potential of width $a_{x}$ and a square well of depth $a_{z}$, approximate the charge in the wire as being uniformly distributed over an ellipse with $a_{x}$ and $a_{z}$ as the principal axes. Define elliptic coordinates $(u, v)$ in the $(x, z)$ plane as

$$
\begin{aligned}
& x=b \cosh (u) \cos (v), \\
& z=b \sinh (u) \sin (v)
\end{aligned}
$$

in which $b=\sqrt{a_{x}^{2}-a_{z}^{2}}$, so that the ellipse corresponds to $u=u_{e}=\cosh ^{-1}\left(a_{x} / b\right)$. As an approximation to the potential for an elliptical charge, use

$$
\tilde{\Phi}= \begin{cases}\alpha u-\gamma & \text { for } u>u_{e} \\ \beta\left(a_{x} x^{2}+a_{z} z^{2}\right)-\kappa & \text { for } u<u_{e}\end{cases}
$$

In the limit $u \rightarrow \infty, u \approx \log (r / c)$, which implies that $\alpha=\bar{\rho} / 2 \pi$ in which $\bar{\rho}$ is the total charge on the ellipse. At the top of the layered material, the correction $\tilde{\Phi}$ should vanish. Apply this at the value $u_{0}=u(z=0, x=0)$ to get $\gamma=u_{0} \bar{\rho} / 2 \pi$. The total charge in the wire $\bar{\rho}$ is approximately given by

$$
\bar{\rho}=\pi^{-1} \sqrt{d \lambda^{w} / c}\left((2 / 3) N^{3 / 2}+N\right)
$$

in which $c=\frac{\hbar^{2}}{2 m}$ and $N=-\lambda_{1}^{w} / d \lambda^{w}$ is the number of transverse eigenvalues that are less than the Fermi energy. Formula (3.30) comes from the number of longitudinal states below the Fermi energy for each transverse state. The potential corrections in (3.29) are used to correct the eigenvalue $\lambda_{1}^{w}$ and eigenvalue spacing $d \lambda^{w}$, which are then used in (3.30). These two equations are solved iteratively to determine $\bar{\rho}$. 


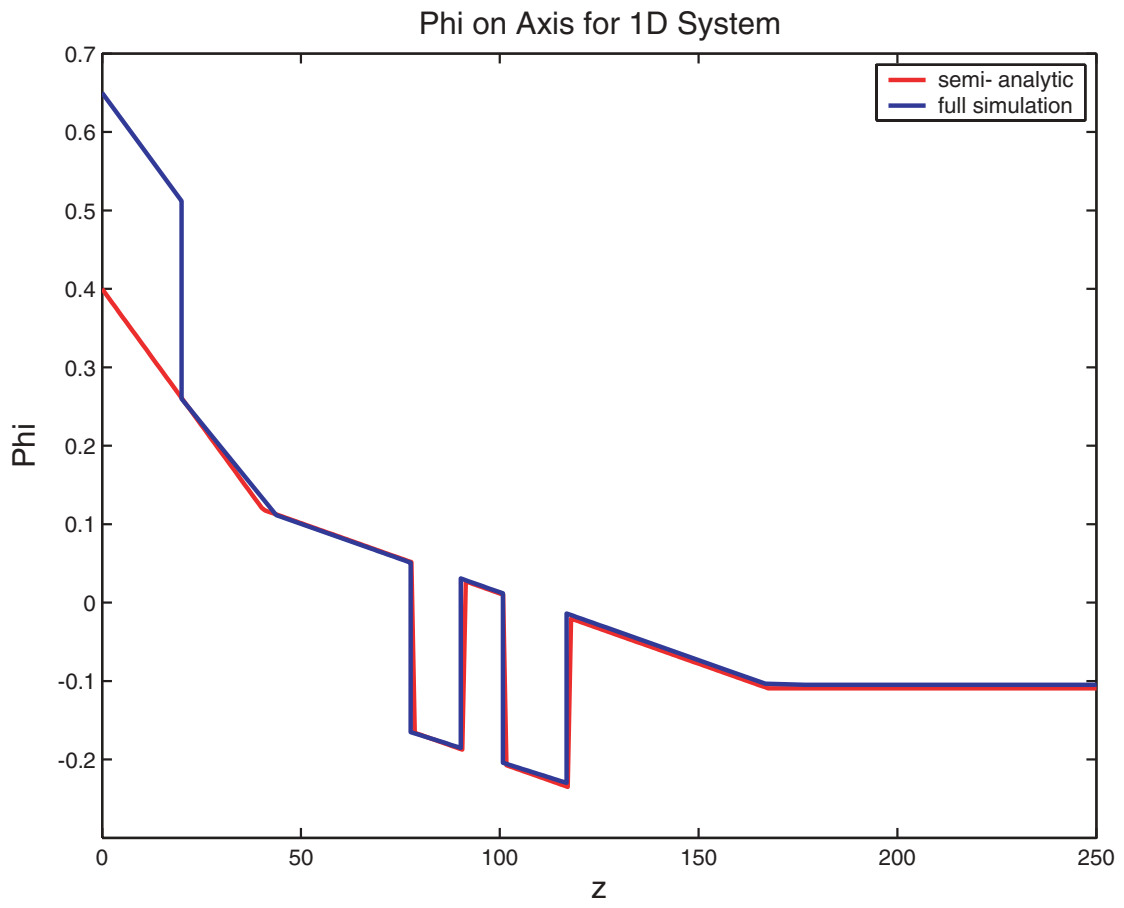

FIG. 3. Comparison of potential $\Phi$ for a one-dimensional geometry from the full scale simulation method (blue) and the semianalytic model (red), with no top gates, no Coulomb interactions, selfconsistent effects omitted, and no background doping, showing excellent agreement. Disagreement at the top is due to use of a different material layer in the full-scale simulation that does not influence the potential outside that layer and was not included in the semianalytic model.

4. Validation of semianalytic model. Validation of the semianalytic model is performed by comparison to a full-scale numerical solution of the Schrödinger-Poisson equations by Anderson [2]. Consider a system with design parameters

$$
\begin{aligned}
v_{d} & =\left(d z_{1}, d z_{2}, d z_{3}, d z_{4}, d z_{5}, d z_{6}, R_{g}, R_{b}, \sigma_{1}, \sigma_{2}\right) \\
& =\left(40.5,37.1,12.6,10.6,16,50.7,61,219.5,3.6 \times 10^{11}, 1.25 \times 10^{11}\right)
\end{aligned}
$$

and with operation parameters $v_{o}=\left(\phi_{g}, \phi_{b}\right)=(0,0.53)$. Figures 3,4 , and 5 show the potential on the central line (through the center of the quantum dot) in one, two, and three dimensions, respectively, with no Coulomb interactions, self-consistent effects omitted, and no background doping. In one dimension there are no gates on the top of the system, so that the potential $\Phi$ is a function of $z$ only. In two dimensions the central gate is an interval (i.e., a strip in three dimensions); while in three dimensions the central gate is a circular dot. Figure 6 is the same as Figure 4, except that self-consistent effects are included in both the full-scale numerical computation and the semianalytic model. The first eigenvalue is shown for each of these problems in Table 3, in $m e V$.

These results show excellent agreement for the case with no Coulomb interactions and no self-consistent effects. In this case the energy errors in the semianalytic method are all within $6 \mathrm{meV}$ of those for the full simulation. With self-consistent effects, the agreement is still good, with energy errors of size $20 \mathrm{meV}$. 


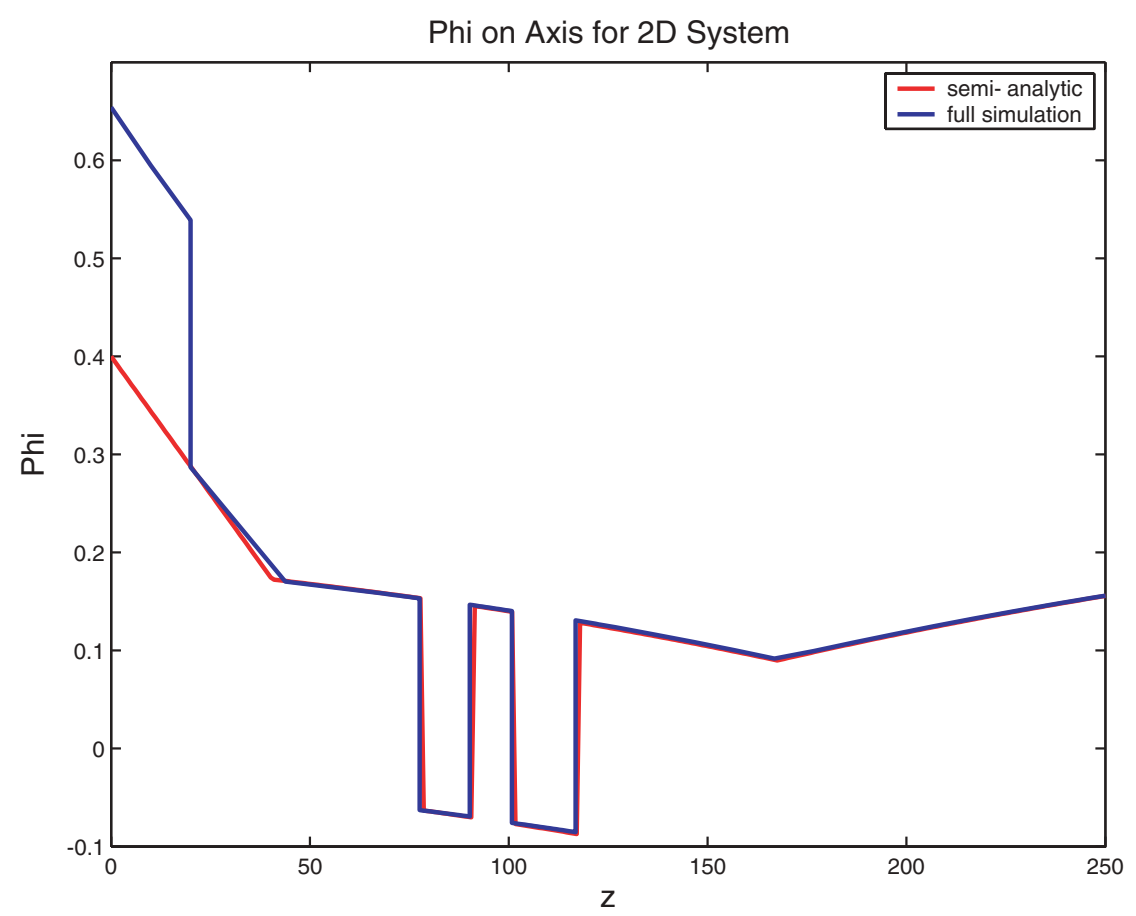

FIG. 4. Same as Figure 3 except that the plot is for the potential $\Phi$ on the central axis $x=0$ for a two-dimensional geometry.

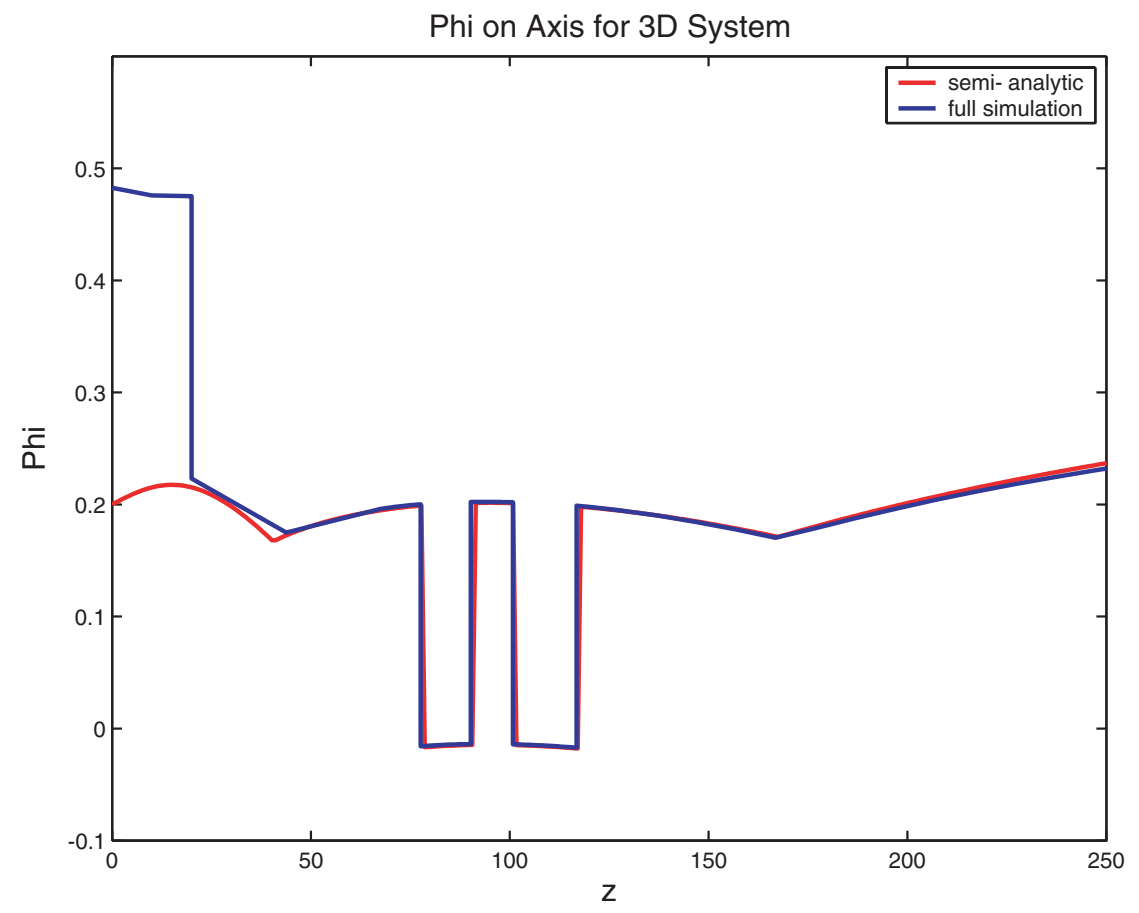

Fig. 5. Same as Figure 3 except that the plot is for the potential $\Phi$ on the central axis $r=0$ for a three-dimensional geometry. 


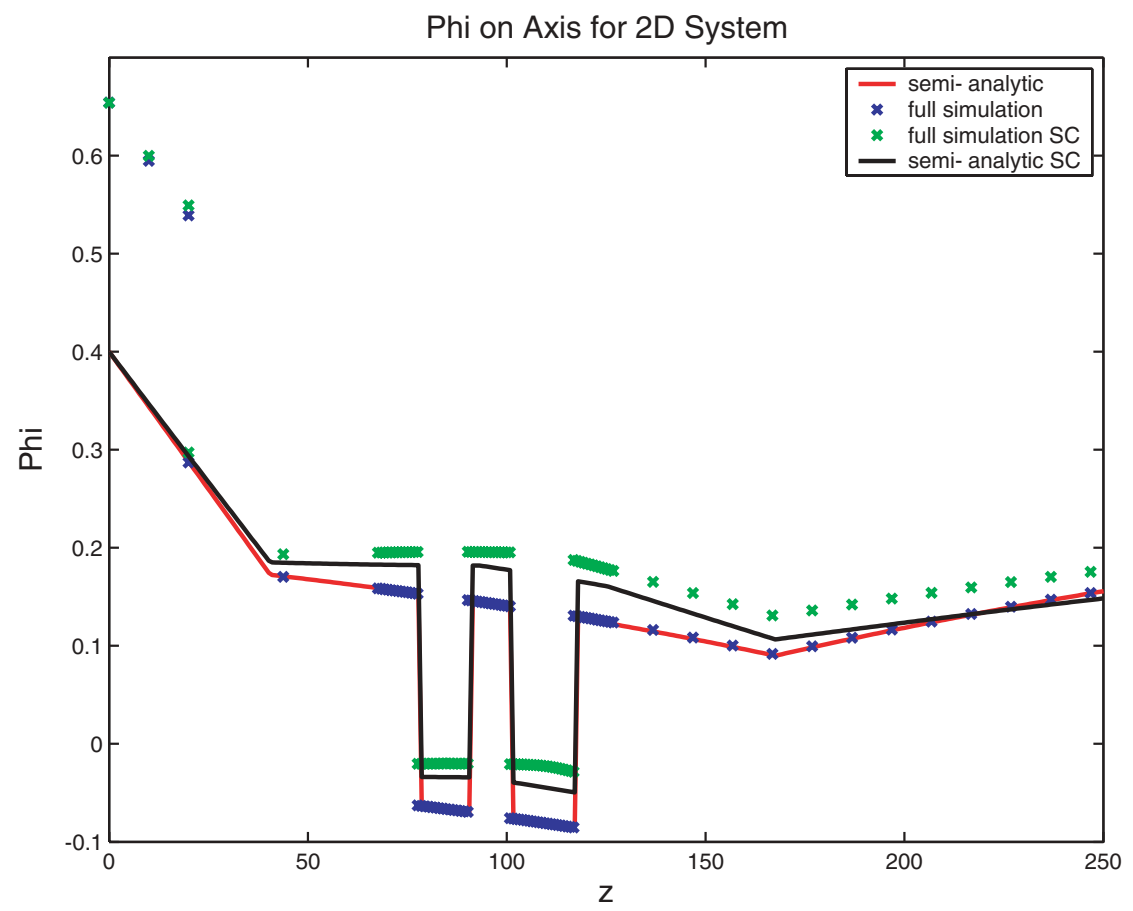

FIG. 6. Same as Figure 4 but showing the full-scale simulation method with (green) and without (blue) self-consistent terms and the semianalytic model with (black) and without (red) self-consistent terms.

TABLE 3

Lowest eigenvalues $\lambda_{1}$.

\begin{tabular}{||l|ll|ll||}
\hline & \multicolumn{2}{|c|}{ Dot energies $(\mathrm{meV})$} & \multicolumn{2}{c||}{ Wire energies $(\mathrm{meV})$} \\
\hline & simulation & semianalytic & simulation & semianalytic \\
1D & -144.1 & -148.3 & -195.9 & -201.1 \\
2D & -33.8 & -37.3 & -58.8 & -60.8 \\
3D & 22.5 & 16.6 & 9.88 & 7.1 \\
2D SC & 11.6 & -4.7 & -3.7 & -23.3 \\
\hline
\end{tabular}

5. Double pinchoff designs. The principal virtue of the semianalytic model is that the eigenvalues in the design criteria (2.15) and (2.16) can be quickly computed, enabling rapid throughput as required for a design study or optimization exercise. In this section we describe a design that achieves the strictest design criterion (2.15) and (2.16) with $k=1$, i.e., double pinchoff with a single electron in the dot and a single conductive state in the wire.

An example of a system achieving double pinchoff has design parameters

$$
\begin{aligned}
v_{d} & =\left(d z_{1}, d z_{2}, d z_{3}, d z_{4}, d z_{5}, d z_{6}, R_{g}, R_{b}, \sigma_{1}, \sigma_{2}\right) \\
& =\left(40,94.3,22.5,30.3,17.7,50,53.7,876.7,3.4 \times 10^{11}, 1.4 \times 10^{11}\right)
\end{aligned}
$$

and operation parameters $v_{o}=\left(\phi_{g}, \phi_{b}\right)=(-0.16,1.47)$. The eigenvalues for this design are $\left(\lambda_{1}^{d}, \lambda_{2}^{d}, \lambda_{1}^{w}(0), \lambda_{2}^{w}\right)=(-0.67,2.2,-0.92,0.27) \mathrm{meV}$. This system was found by a random search over the full space of possible design parameters and operation 
parameters. In a search involving 10 million trial designs, 7 successful designs were found. This establishes existence (within the simulation) of a design meeting the double pinchoff goal. In section 7 , however, we show that these double pinchoff designs are not robust with respect to fabrication errors.

6. Design robustness. When a prescribed design is implemented, the outcome will differ from the prescription due to errors and uncertainties in growth and fabrication, including variability in the layer thicknesses and gate sizes and variability in charge density in delta-doped layers due to uncertainties in both the doping level and the ionization fraction. Additional modeling uncertainties, such as uncertainties in the correct boundary conditions at the top of the device and additional physics such as self-consistent terms, are not accounted for in this analysis.

To find a design whose success is insensitive to the growth and fabrication uncertainties, we formulate a measure of design robustness. Assume that the errors in each of the various design parameters are independent and normally distributed and define $\alpha_{k}$ to be the standard deviation of the $k$ th design parameter. Define a distance function $d$ between two design vectors $v$ and $w$ as

$$
d(v, w)=\left(\sum_{k=1}^{K}\left(\left(v_{k}-w_{k}\right) / \alpha_{k}\right)^{2}\right)^{1 / 2},
$$

i.e., $d(v, w)$ is a measure of the distance between $v$ and $w$ in standard deviations. Next fix a design criterion by choosing the number $K$ of allowed conduction states in the wire, and define the robustness $R$ of a successful design $v_{s}$ as the distance to the nearest unsuccessful design $v_{u}$, i.e.,

$$
R\left(v_{s}\right)=\min _{v_{u}} d\left(v_{s}, v_{u}\right) .
$$

The design robustness optimization problem is to find the most robust design within the constraint set $C$ from (2.1)-(2.6), i.e., $v_{s}$ is chosen to be the successful design that achieves the following max-min:

$$
\max _{v_{s} \in C} R\left(v_{s}\right)=\max _{v_{s} \in C} \min _{v_{u} \in C} d\left(v_{s}, v_{u}\right) .
$$

As an example for standard deviation of the fabrication, we take standard deviation of the growth processes (layer thicknesses) to be $3 \%$ (relative error), the standard deviation of the fabrication (gate sizes) to be $10 \mathrm{~nm}$ (absolute error), and the standard deviation of the charge density in the delta-doped layers to be $40 \%$ (relative error).

7. Analysis of failure modes. A direct random search for the design $v_{s}$ that achieves the max-min in (6.3) would involve a double random search over two designs $v_{s}$ and $v_{u}$. This can be considerably improved by analysis of the failure modes, i.e., the closest failed designs $v_{u}$ for a given successful design $v_{s}$. This analysis relies on a linear approximation for the dependence of the eigenvalues $\lambda$ in the design criteria (2.15) and (2.16), as functions of the gate voltages $\phi_{g}$ and $\phi_{b}$.

A successful design $v_{s}$ is one for which the four design inequalities in (2.15) and (2.16) form a quadrilateral (or triangular) set that has a nonempty intersection with the rectangular constraint set defined by (2.5) and (2.6), in the operation space $\left(\phi_{g}, \phi_{b}\right)$. As the design parameter vector $v$ is (smoothly) varied, the sides of the quadrilateral (or triangle) will (smoothly) vary. The first unsuccessful design $v_{u}$ 
is reached when the intersection becomes just a point. This characterizes the design $v_{u}$ that occurs in (6.3).

The intersection of the operation window (i.e., the quadrilateral or triangle defined by (2.15) and (2.16)) and the constraint set (defined by (2.5) and (2.6)) can shrink to a point in either of two ways: First, the operation window can shrink to a point in the interior of the constraint set. Second, the operation window can move outside the constraint set with one vertex on the boundary of the constraint set.

We draw the operation window with coordinates $\left(-\phi_{g}, \phi_{b}\right)$, so that both coordinates are positive. Denote the boundaries of the operation window as follows:

$$
\begin{aligned}
& a=\left\{\left(-\phi_{g}, \phi_{b}\right): \lambda_{1}^{d}\left(\phi_{g}, \phi_{b}\right)=0\right\}, \\
& b=\left\{\left(-\phi_{g}, \phi_{b}\right): \lambda_{2}^{d}\left(\phi_{g}, \phi_{b}\right)=0\right\}, \\
& c=\left\{\left(0, \phi_{b}\right): \lambda_{1}^{w}\left(0, \phi_{b}\right)=0\right\}, \\
& d=\left\{\left(-\phi_{g}, \phi_{b}\right): \lambda_{2}^{w}\left(\phi_{g}, \phi_{b}\right)=0\right\} .
\end{aligned}
$$

Also denote $a c$ to be the point of intersection of the lines $a$ and $c$ if it exists, with coordinates $\phi_{g}(a c)$ and $\phi_{b}(a c)$, and similarly for the other intersections. Also denote $0 a$ to be the intersection of $a$ with the line $\phi_{g}=0$. They have the following properties:

1. $c$ is a horizontal line.

2. $a, b$, and $d$ are lines with positive slope, with $a$ and $b$ steeper than $d$.

3. $a$ and $b$ cannot intersect (for $-\phi_{g}>0$ ) and $a$ is to the left of $b$.

4. $-\phi_{g}(a c)<-\phi_{g}(b c)$.

5. The operation window is nonempty if and only if $-\phi_{g}(a c)<-\phi_{g}(c d)$.

6. ad is the leftmost and the lowest point of the operation window.

From these properties, it follows that a nonempty operation window can have two possible configurations. If $-\phi_{g}(b c)<-\phi_{g}(c d)$, it is a quadrilateral with vertices $a d, b d, b c$, and $a c$, which is denoted as Type I. If $-\phi_{g}(b c)>-\phi_{g}(c d)$, a nonempty operation window is a triangle with vertices $a d, c d$, and $a c$, which is denoted as Type II. These two possibilities are shown in Figure 7.
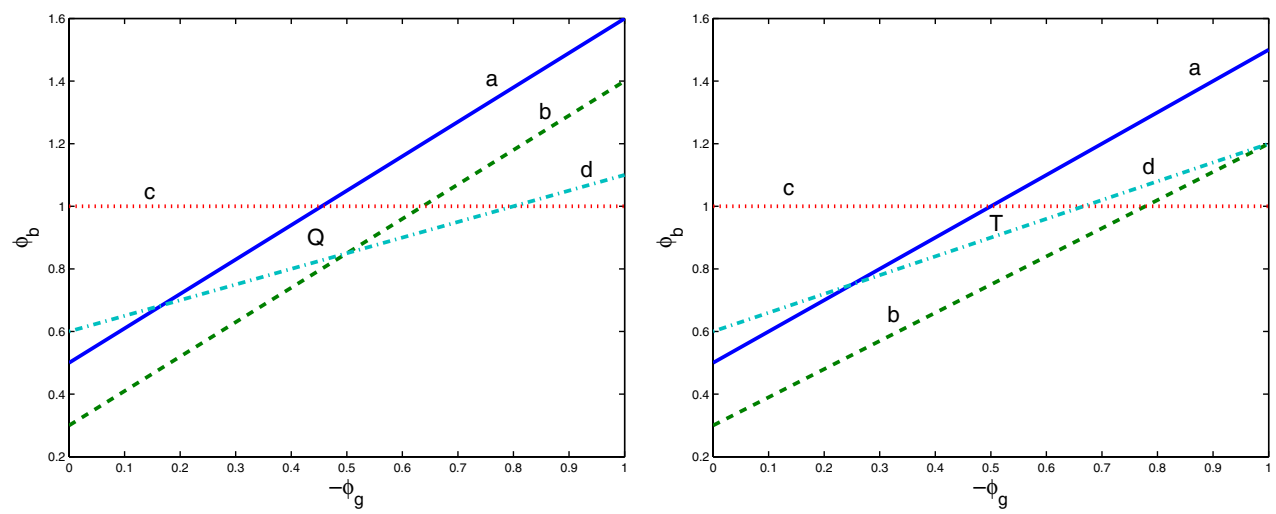

FIG. 7. A schematic drawing of the operation windows in $\left(-\phi_{g}, \phi_{b}\right)$. The four lines that define the operation window are $a, b, c$, and $d$. The successful operation vectors are those in quadrilateral region labeled $Q$ for the configuration on the left or in the triangular region labeled $T$ for the configuration on the right. 
This information allows characterization of the failure modes:

A Collapse of the operation window can occur only as a transition from Type II, in which the three vertices of the triangle meet as one point acd. Failure mode $\mathrm{A}$ is characterized by existence of a triple intersection point $a c d$, which is denoted as point $A$.

B If the operation window leaves the constraint region through the upper boundary, $\phi_{b}=\bar{\phi}_{b}$, then the final point of intersection of the two regions is ad. Failure mode $\mathrm{B}$ is characterized by existence of a triple intersection point ad with $\phi_{b}=\bar{\phi}_{b}$, which is denoted as point $B$.

$\mathrm{C}$ If the operation window leaves the constraint region through the right boundary, $-\phi_{g}=\bar{\phi}_{g}$, then the final point of intersection of the two regions is ad. Failure mode $\mathrm{C}$ is characterized by existence of a triple intersection point $a d$ with $-\phi_{g}=\bar{\phi}_{g}$, which is denoted as point $C$.

D If the operation window leaves the constraint region through the lower boundary, denoted as failure mode $\mathrm{D}$, then line $c$ coincides with the lower constraint $\phi_{b}=\phi_{b}$.

E If the operation window leaves the constraint region through the left boundary, $-\phi_{g}=\underline{\phi}_{g}$, then the final point of intersection of the two regions is $b c$ in Type I or $c d$ in Type II. Failure mode $\mathrm{E}$ is characterized by existence of a triple intersection point $b c$ or $c d$ with $-\phi_{g}=\underline{\phi}_{g}$, which is denoted as point $E$. The three failure points $A, B$, and $C$, which are the ones that most frequently occur, are illustrated in Figure 8
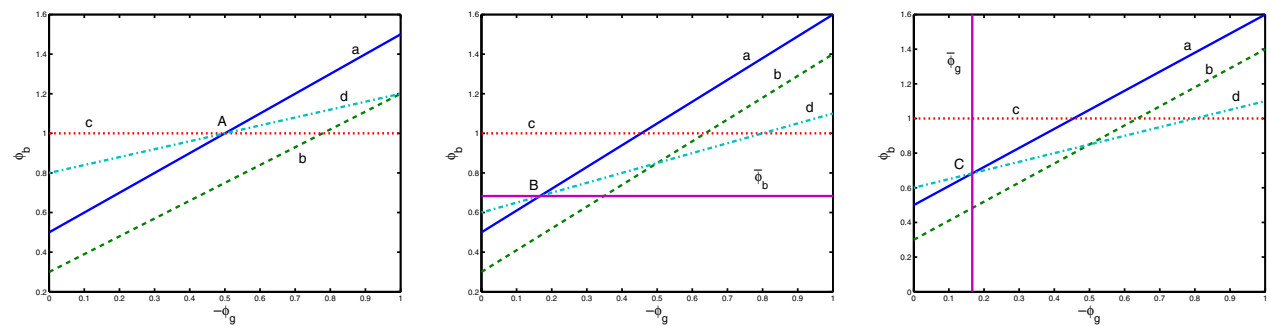

FIG. 8. A schematic drawing of the failure modes in $\left(-\phi_{g}, \phi_{b}\right)$, including the four lines $a, b$, $c$, and $d$ that define the operation window, and the constraining lines $\phi_{b}=\bar{\phi}_{b}$ in the middle and $-\phi_{g}=\bar{\phi}_{g}$ in the right. In mode $A$ (left), the operation window has collapsed to a point $A$ in the interior of the constraint set. In mode $B$ (middle), the operation window intersects the constraint set in only a single point $B$ on the upper boundary. In mode $C$ (right), the operation window intersects the constraint set in only a single point $C$ on the right boundary.

The distance from a successful design $v_{s}$ to one of the failure points $A, B$ or $C$ can be estimated through a linear approximation. For point $A$, let $\left(\Phi_{g}^{A}(v), \Phi_{b}^{A}(v)\right)$ solve

$$
\begin{aligned}
\lambda_{1}^{d}\left(\Phi_{g}^{A}(v), \Phi_{b}^{A}(v), v\right) & =0, \\
\lambda_{1}^{w}\left(0, \Phi_{b}^{A}(v), v\right) & =0 .
\end{aligned}
$$

Then to leading order, since $\lambda_{2}^{w}\left(\Phi_{g}^{A}(A), \Phi_{b}^{A}(A), A\right)=0$,

$$
\begin{aligned}
\lambda_{2}^{w}\left(\Phi_{g}^{A}(v), \Phi_{b}^{A}(v), v\right) & =\lambda_{2}^{w}\left(\Phi_{g}^{A}(v), \Phi_{b}^{A}(v), v\right)-\lambda_{2}^{w}\left(\Phi_{g}^{A}(A), \Phi_{b}^{A}(A), A\right) \\
& =(v-A) \cdot \nabla_{v} \lambda_{2}^{w}\left(\Phi_{g}^{A}(v), \Phi_{b}^{A}(v), v\right) .
\end{aligned}
$$


At a minimum point, the design difference $v-A$ is parallel to the gradient in (7.7), so that

$$
\min |v-A|=\left|\lambda_{2}^{w}\left(\Phi_{g}^{A}(v), \Phi_{b}^{A}(v), v\right)\right| /\left|\nabla_{v} \lambda_{2}^{w}\left(\Phi_{g}^{A}(v), \Phi_{b}^{A}(v), v\right)\right| .
$$

A similar analysis can be carried out for $B$ and $C$. For $B, \Phi_{b}^{B}(v)=\bar{\phi}_{b}$ and let $\Phi_{g}^{B}(v)$ solve

$$
\lambda_{1}^{d}\left(\Phi_{g}^{B}(v), \bar{\phi}_{b}, v\right)=0 .
$$

Then to leading order, since $\lambda_{2}^{w}\left(\Phi_{g}^{B}(B), \bar{\phi}_{b}, B\right)=0$, it follows that

$$
\min |v-B|=\left|\lambda_{2}^{w}\left(\Phi_{g}^{B}(v), \bar{\phi}_{b}, v\right)\right| /\left|\nabla_{v} \lambda_{2}^{w}\left(\Phi_{g}^{B}(v), \bar{\phi}_{b}, v\right)\right| .
$$

For $C, \Phi_{g}^{C}(v)=\bar{\phi}_{g}$ and let $\Phi_{b}^{C}(v)$ solve

$$
\lambda_{1}^{d}\left(\bar{\phi}_{g}, \Phi_{g}^{C}(v), v\right)=0 .
$$

Then to leading order, since $\lambda_{2}^{w}\left(\bar{\phi}_{g}, \Phi_{g}^{C}(C), C\right)=0$, it follows that

$$
\min |v-C|=\left|\lambda_{2}^{w}\left(\bar{\phi}_{g}, \Phi_{g}^{C}(v), v\right)\right| /\left|\nabla_{v} \lambda_{2}^{w}\left(\bar{\phi}_{g}, \Phi_{g}^{C}(v), v\right)\right| .
$$

The robustness $R$ and the design robustness optimization problem can now be rephrased as

$$
\begin{array}{r}
R\left(v_{s}\right)=\min \{|v-A|,|v-B|,|v-C|\}, \\
\max _{v_{s} \in C} R\left(v_{s}\right)=\max _{v_{s} \in C} \min \{|v-A|,|v-B|,|v-C|\}
\end{array}
$$

in which $|v-A|,|v-B|$, and $|v-C|$ are defined by (7.8), (7.10), and (7.12). This has the advantage over the formulation (6.3) that it requires only a single random search for successful designs $v_{s}$ rather than a double random search for $v_{s}$ and $v_{d}$. For each $v_{s}$, the min is found by evaluation of the three quantities $|v-A|,|v-B|$ and $|v-C|$ from (7.8), (7.10), and (7.12).

For the design $v_{d p}$ that achieved double pinchoff, as described in section 5 , the design robustness distance (from (7.13)) is $R\left(v_{d p}\right)=0.3$, which corresponds to probability of about 0.2 of successful design. The search for a more robust design through maximization of $R\left(v_{s}\right)$ as in (7.14) is described in section 8 .

8. Design optimization. The search for a maximally robust design $v_{s}$ in (7.14) can be accelerated by decomposition and some analysis. First select values for the geometrical design parameters $v^{\prime}=\left(d z_{1}, d z_{2}, d z_{3}, d z_{4}, d z_{5}, d z_{6}, R_{g}, R_{b}\right)$.

For a given choice of geometrical parameters $v^{\prime}$, the possible values of the $\delta$-doping densities $\sigma_{1}$ and $\sigma_{2}$ can be determined using the linear dependence of the eigenvalues $\lambda_{1}^{d}$ and $\lambda_{1}^{w}$ on $\sigma_{1}$ and $\sigma_{2}$, as well as on $\phi_{g}$ and $\phi_{b}$. The operation window can be characterized as having the point $a c$ inside the constraint set. The two equations (7.1) and (7.3) defining $a c$ can be used to define a mapping between the operation vector $\left(\phi_{g}, \phi_{b}\right)$ and the density vector $\left(\sigma_{1}, \sigma_{2}\right)$. Then the constraint set defined by (2.5) and (2.6) can be mapped to a constraint set in the space of density vectors, which may need to be cut off to accommodate the constraints (2.4). To simplify, we choose a value of $\left(\phi_{g}, \phi_{b}\right)$ that is approximately in the center of the resulting polygon. This is illustrated in Figure 9. 


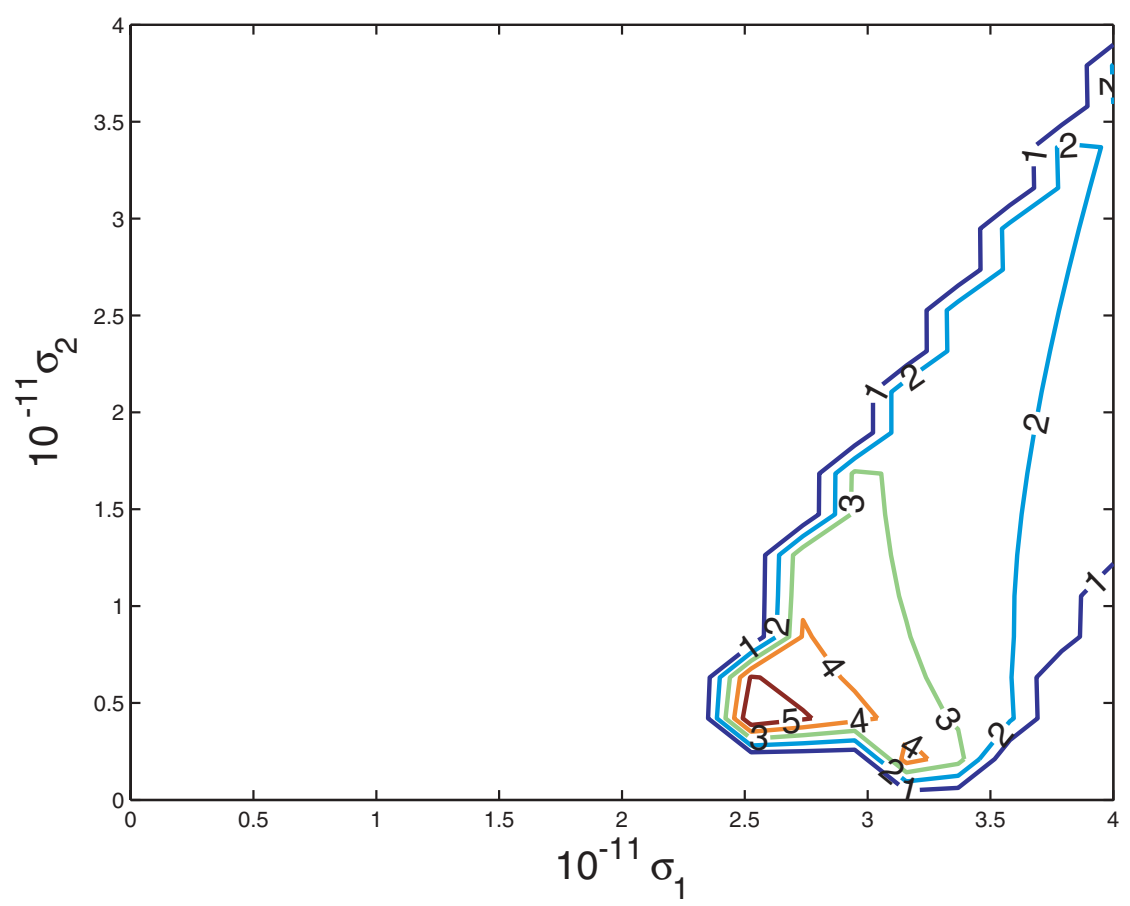

FIG. 9. Design window in the space of doping densities $\left(\sigma_{1}, \sigma_{2}\right)$ for one of the optimally robust designs.

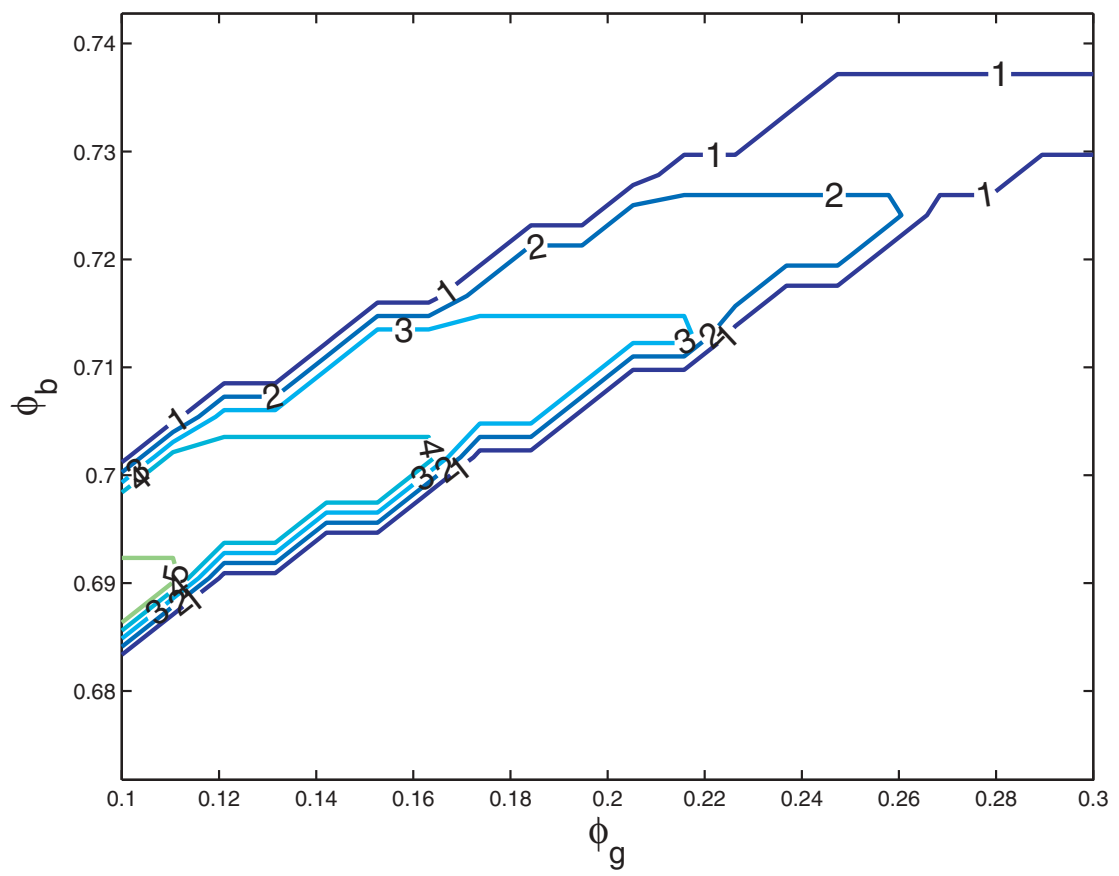

FIG. 10. Design window in the space of operation vectors $\left(-\phi_{g}, \phi_{b}\right)$ for one of the optimally robust designs. 
Following this procedure for $K=7$, we have found designs with robustness values of 2.5 or more. A typical result is

$$
\text { (8.1) } \begin{aligned}
v & =\left(d z_{1}, d z_{2}, d z_{3}, d z_{4}, d z_{5}, d z_{6}, R_{g}, R_{b}, \sigma_{1}, \sigma_{2}\right) \\
& =\left(47.25,49.31,8.24,16,23.62,50,50.38,608.28,3.78 \times 10^{11}, 2.45 \times 10^{11}\right) .
\end{aligned}
$$

The operation vector is $\left(\phi_{g}, \phi_{b}\right)=(0.24,1.91)$. The eigenvalues for this design are $\left(\lambda_{1}^{d}, \lambda_{2}^{d}, \lambda_{1}^{w}(0), d \lambda^{w}\right)=(-0.559,3.60,-33.0,4.0)$. There are seven transverse states in the wire and the robustness is 2.8 , which corresponds to more than $99 \%$ probability of a successful design. The resulting operation window is shown in Figure 10.

9. Conclusions. In this work, we have developed a mathematical model for an electron spin qubit system, for the successful design of the system, and for optimization of the design robustness. In addition, we have developed a simple semianalytic model that is both sufficiently accurate to provide relevant results for the system and sufficiently fast to allow for the high throughput required by design and optimization studies. After some analysis to simplify the computation of design robustness, we have performed a random search for designs that satisfy the design criteria and for designs that are maximally robust.

From this search, we have found system designs that achieve double pinchoff, in the sense that they have a single electron in the quantum dot and a single conduction state in the quantum wire. These designs are not sufficiently robust to be practical, having a design robustness of only about 0.3 , in terms of standard deviation using a current assessment of design uncertainties. By relaxing the design criterion to allow for a small number (e.g., $K=7$ ) of conduction states in the wire, we have found designs that are more than 2.8 standard deviations from an unsuccessful design. Currently these designs are being built and tested for their electronic properties.

Several conclusions can be drawn from the present study. First is the importance of models at different levels of complexity. A full-scale model, as in [2], is needed to give reliable values for the system properties and to provide validation for simpler models. Simpler models that are less computationally intensive are also needed, however, to enable design and optimization studies on a reasonable time scale. In addition, we have been using nextnano ${ }^{3}$ [13], a computational physics software package, which includes a much wider set of physics in order to check and validate the results from full-scale numerical solver for the Schrödinger-Poisson equation [2]. Second is the importance of analysis as a method for accelerating the random search that is often required in a design and optimization study. In the present study, the search for an optimally robust design was greatly aided by analysis of the failure modes for a design (i.e., the closest unsuccessful designs to a given successful design) and elimination of the charge variables using their special (linear) occurrence in the model.

\section{REFERENCES}

[1] G. Burkard, D. Loss, and D. P. DiVincenzo, Coupled quantum dots as quantum gates, Phys. Rev. B, 59 (1999), pp. 2070-2078.

[2] C. R. ANDERson, private communication, 2004.

[3] I. A. Fedorov, K. W. Kim, R. E. Caflisch, and E. Yablonovitch, A Scalable Quantum Gate Design for Quantum Computation, preprint, 2004.

[4] T. Fujisawa, D. G. Austing, Y. Tokura, Y. Hirayama, and S. Tarucha, Allowed and forbidden transitions in artificial hydrogen and helium atoms, Nature, 419 (2002), pp. $278-281$.

[5] M. Fujiwara, M. Takeoka, J. Mizuno, and M. Sasaki, Exceeding classical capacity limit in quantum optical channel, Phys. Rev. Lett., 90 (2003), 167906. 
[6] L. K. Grover, Quantum mechanics helps in searching for a needle in a haystack, Phys. Rev. Lett., 79 (1997), pp. 325-328.

[7] M. Gyure, private communication.

[8] X. Hu AND S. DAS SARMA, Hilbert-space structure of a solid-state quantum computer: Twoelectron states of a double-quantum-dot artificial molecule, Phys. Rev. A, 61 (2000), 062301.

[9] A. Imamoglu, D. D. Awschalom, G. Burkard, D. P. DiVincenzo, D. Loss, M. Sherwin, AND A. Small, Quantum Information Processing Using Quantum Dot Spins and Cavity QED, Phys. Rev. Lett., 83 (1999), pp. 4204-4207.

[10] B. E. KANE, A silicon-based nuclear spin quantum computer, Nature, 393 (1998), pp. 133-137.

[11] J. LEVY, Quantum-information processing with ferroelectrically coupled quantum dots, Phys. Rev. A, 64 (2001), 052306.

[12] D. Mozyrsky, V. Privman, and M. L. Glasser, Indirect interaction of solid-state qubits via two-dimensional electron gas, Phys. Rev. Lett., 86 (2001), pp. 5112-5115.

[13] nextnano ${ }^{3}$ : Next Generation 3D Nano Device Simulator. http://www.wsi.tum.de/nextnano3/.

[14] M. A. Nielsen And I. L. ChuAng, Quantum Computation and Quantum Communication, Cambridge University Press, Cambridge, UK, 2000.

[15] P. W. SHor, Polynomial-time algorithms for prime factorization and discrete logarithms on a quantum computer, SIAM J. Comput., 26 (1997), pp. 1484-1509.

[16] S. Somaroo, C. H. Tseng, T. F. Havel, R. Laflamme, and D. G. Cory, Quantum simulations on a quantum computer, Phys. Rev. Lett., 82 (1999), pp. 5381-5384.

[17] R. Vrijen, E. Yablonovitch, K. L. Wang, H. W. Jiang, A. Balandin, V. Roychowdhury, T. MoR, AND D. DiVINCEnzo, Electron spin resonance transistors for quantum computing in silicon-germanium hetero-structures, Phys. Rev. A, 62 (2000), 012306.

[18] L.-X. Zhang, P. Matagne, J. P. Leburton, R. Hanson, and L. P. Kouwenhoven, Singleelectron charging and detection in a laterally coupled quantum-dot circuit in the fewelectron regime, Phys. Rev. B, 69 (2004), 245301. 